\title{
Research on Ranging Optimization and Twice-Weighted Centroid Calculation in Wireless Sensor Network Localization Algorithm
}

\author{
Leina Gao* and Jianshe Peng
}

School of Industrial Manufacturing, Chengdu University, Chengdu, Sichuan 610106, China

\begin{abstract}
In practical applications of ranging technology commonly used in wireless sensor positioning algorithm, ranging between nodes will inevitably produce errors due to environmental factors and measurement hardware effects. The wireless sensor network localization algorithm DGC-TWCL proposed in this paper takes advantage of the Cayley-Menger determinant used to provide distance geometry constraints(DGC) in two-dimensional real space to optimize and correct the ranging error, combined with the twice-weighted centroid localization(TWCL) calculation, the final position of the unknown node can be obtained. The experiment results show that the algorithm can get the positioning results with higher accuracy and better robustness.
\end{abstract}

Keywords: Localization, wireless sensor network, inaccurate ranging, Cayley-Menger determinant, twice weighted centroid localization computation.

\section{INTRODUCTION}

As an international hot research field attracting a great deal of attention nowadays, wireless sensor network (WSN) is a new pattern of information acquisition and processing. The networked computing devices with low price and low power provide a novel design mode to the connectivity between the physical world and human society in ubiquitous era. The node localization is one of the most important issues after many WSN system layouts have been complete. The monitoring data for many applications with unknown location have no meaning. In usual case, WSN positioning algorithm obtains the unknown node position estimation through measurement or connection between the unknown node and anchor nodes with priori position information. According to the current status, WSN positioning technology can be divided into ranging-based and non-ranging-based in view of whether the metrics between two nodes is necessary during localization process. The former positioning mode has relatively higher accuracy and cost, while the latter based on the connectivity of network has less accuracy and hardware requirement. In accordance with hops between the unknown node and anchor nodes in connection, the localization algorithm is divided into single-hop and multi-hops. From the viewpoint of processing manner to the measurement data among nodes, WSN positioning can be divided into the centralized and distributed method. The centralized algorithm uses the central processor to get all ranging information resulting in the entire network location mapping and the distributed algorithm achieves self-positioning by node acquisition of local information to the neighbor nodes. The typical

*Address correspondence to this author at the School of Industrial Manufacturing, Chengdu University, Chengdu, Sichuan 610106, China;

Tel: +086-028-84616075; Fax: +086-028-84616075;

Email: leinna@163.com location algorithms and systems include centroid method [1], least squares estimation method [2], the MIT Criket [3], the convex programming location algorithm of the University of California, Berkeley [4], DV-Hop [5] and DV-Distance [6] distributed localization algorithm of the Rutgers University.

The common ranging technology of WSN localization algorithm includes RSS, AoA [7], ToA and TDoA[8]. In practical application environments, ranging has the errors inevitably because of the impact of multi-path, reflection, diffraction, scattering and other environment or measurement hardware factors. Since the distance between nodes is independent from each other, the ranging error may undermine geometric topology relationship e.g. the trilateration measurements make the length of both sides less than the third one, which causes failure of the localization algorithm. This paper proposes a wireless sensor network localization algorithm DGC-TWCL which utilizes Cayley-Menger determinant in two-dimension real space to provide distance geometry constraints(DGC), makes optimization and correction to the ranging errors and obtains the final position of the unknown node combined with the twice-weighted centroid localization(TWCL) calculation.

\section{CAYLEY-MENGER DETERMINANT AND OPTI- MIZATION OF DISTANCE GEOMETRY CON- STRAINTS}

\subsection{Cayley-Menger Determinant}

In the theory of distance geometry, Cayley-Menger determinant can be used to handle the geometry problem of Euler distance in invariant space [9]. Two n-points sequences $\left\{\mathrm{p}_{1}, \mathrm{p}_{2}, \ldots, \mathrm{p}_{\mathrm{n}}\right\}$ and $\left\{\mathrm{q}_{1}, \mathrm{q}_{2}, \ldots, \mathrm{q}_{\mathrm{n}}\right\}$ in m-dimension real space form the Cayley-Menger matrix which is defined as: 


$$
\begin{aligned}
& M\left(p_{1}, \ldots, p_{n} ; q_{1}, \ldots, q_{n}\right) \\
& \triangleq\left[\begin{array}{ccccc}
d^{2}\left(p_{1}, q_{1}\right) & d^{2}\left(p_{1}, q_{2}\right) & \cdots & d^{2}\left(p_{1}, q_{n}\right) & 1 \\
d^{2}\left(p_{2}, q_{1}\right) & d^{2}\left(p_{2}, q_{2}\right) & \cdots & d^{2}\left(p_{2}, q_{n}\right) & 1 \\
\vdots & \vdots & \ddots & \vdots & \vdots \\
d^{2}\left(p_{n}, q_{1}\right) & d^{2}\left(p_{n}, q_{2}\right) & \cdots & d^{2}\left(p_{n}, q_{n}\right) & 1 \\
1 & 1 & \cdots & 1 & 0
\end{array}\right],
\end{aligned}
$$

where $d\left(p_{i}, q_{j}\right), i, j \in\{1, . ., n\}$ is the Euclidean distance between the point $p_{i}$ and $q_{j}$. The bi-determinant of CayleyMenger composed by the two n-point sequences is defined as:

$$
D\left(p_{1}, \ldots, p_{n} ; q_{1}, \ldots, q_{n}\right) \triangleq \operatorname{det} M\left(p_{1}, \ldots, p_{n} ; q_{1}, \ldots, q_{n}\right) .
$$

When the two sequences of points are the same, $M\left(p_{1}, \ldots, p_{n} ; p_{1}, \ldots, p_{n}\right)$ and $D\left(p_{1}, \ldots, p_{n} ; p_{1}, \ldots, p_{n}\right)$ can be defined simply as $M\left(p_{1}, \ldots, p_{n}\right)$ and $D\left(p_{1}, \ldots, p_{n}\right) \cdot D\left(p_{1}, \ldots, p_{n}\right)$ is called Cayley-Menger determinant which has the following classic conclusion:

(Theorem 1[10]) considering n-tuple constituted by $p_{1}, \ldots, p_{n}$ in m-dimension space, the rank of Cayley-Menger matrix $M\left(p_{1}, \ldots, p_{n}\right)$ is at most $m+1$.

According to the above theorem, it's easy to know that in two-dimension real space, $D\left(p_{0}, p_{1}, p_{2}, p_{r}\right)=0 . p_{0}$ can be regarded as the unknown node during the positioning process and it makes the distance measurements with the three known anchor nodes $p_{1}, p_{2}, p_{r}$, which is shown in (Fig. 1).

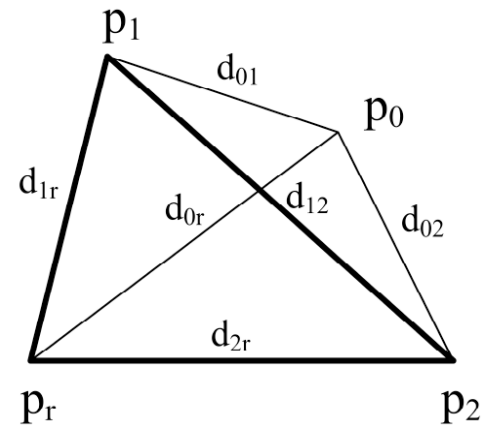

Fig. (1). Four-tuple geometric relationship in two-dimension space.

The value of Cayley-Menger determinant comprised by $d_{01}, d_{02}, d_{0 r}$ and the distances $d_{12}, d_{1 r}, d_{2 r}$ among the anchor nodes is 0 , that is:

$$
D\left(p_{0}, p_{1}, p_{2}, p_{r}\right)=\left|\begin{array}{ccccc}
0 & d_{01}^{2} & d_{02}^{2} & d_{0 r}^{2} & 1 \\
d_{01}^{2} & 0 & d_{12}^{2} & d_{1 r}^{2} & 1 \\
d_{02}^{2} & d_{12}^{2} & 0 & d_{2 r}^{2} & 1 \\
d_{0 r}^{2} & d_{1 r}^{2} & d_{2 r}^{2} & 0 & 1 \\
1 & 1 & 1 & 1 & 0
\end{array}\right|=0 .
$$

Due to noise influence of the environment and equipment, error $\varepsilon$ inevitably occurs during the actual measurement. It's assumed that the accurate distance $d_{0 i}$ and measured distance $\tilde{d}_{0 i}$ between the unknown node and the anchor node have the following relationship:

$$
\tilde{d}_{0 i}^{2}=d_{0 i}^{2}+\varepsilon_{i}, i=1,2, r .
$$

When Eq.(4) is taken into Eq.(3), the following relationship can be obtained:

$\varepsilon^{T} A \varepsilon+\varepsilon^{T} b+c=0$,

where $\varepsilon=\left[\varepsilon_{1}, \varepsilon_{2}, \varepsilon_{r}\right]^{T}$, the matrix $A$, the vector $b$ and $c$ are described by the distances $d_{12}, d_{1 r}, d_{2 r}$ among the known anchor nodes and measured distances $\tilde{d}_{01}^{2 r}, \tilde{d}_{02}, \tilde{d}_{0 r}$.

\subsection{Ranging Optimization of Distance Geometry Con- straints}

If the unknown node $p_{0}$ receives the noise distance measurement information from anchor nodes $p_{1}, \ldots, p_{r}$, in accordance with the collections of nodes $\left\{p_{0}, p_{1}, p_{2}, p_{3}\right\},\left\{p_{0}, p_{1}, p_{2}, p_{4}\right\}, \ldots\left\{p_{0}, p_{1}, p_{2}, p_{r}\right\}, \mathrm{r}-2$ independent quadratic distance constraint equations can be obtained combined with Eq.(3), Eq.(4) and Eq.(5). Each equation can be expressed as $f_{i}\left(\varepsilon_{1}, \varepsilon_{2}, \varepsilon_{i}\right)=0, i=3,4, \ldots, r$, where $\varepsilon_{1}, \varepsilon_{2}, \ldots, \varepsilon_{r}$ are the errors occurred among the unknown node and anchor nodes during the measurement process. By getting the minimized sum of squared errors under the distance constraints limits, the optimization problem can be expressed as:

$$
\begin{aligned}
& \min J=\varepsilon_{1}^{2}+\varepsilon_{2}^{2}+\ldots+\varepsilon_{r}^{2} \\
& \text { s.t. }\left\{\begin{array}{l}
f_{1}\left(\varepsilon_{1}, \varepsilon_{2}, \varepsilon_{3}\right)=0 \\
f_{2}\left(\varepsilon_{1}, \varepsilon_{2}, \varepsilon_{4}\right)=0 \\
\vdots \\
f_{r-2}\left(\varepsilon_{1}, \varepsilon_{2}, \varepsilon_{r}\right)=0
\end{array}\right.
\end{aligned}
$$

Using numerical analysis methods, the optimization solution $\varepsilon_{1}^{*}, \varepsilon_{2}^{*}, \ldots, \varepsilon_{r}^{*}$ can be get. Combined with the Eq.(4), the position estimated between the unknown node and anchor node can be obtained: $\hat{d}_{0 i}=\sqrt{\tilde{d}_{0 i}^{2}-\varepsilon_{i}^{*}}, i=1,2, \ldots, r$.

\section{TWICE-WEIGHTED CENTROID LOCALIZATION COMPUTATION}

Through distance information with the three known anchor nodes, the definite position coordinates of the unknown node in two-dimension space theoretically can be obtained by trilateration measurement method, i.e., three anchor nodes as the centers, the relevant distance information as radiuses of the circles that intersect at one point, as shown in (Fig. 2).

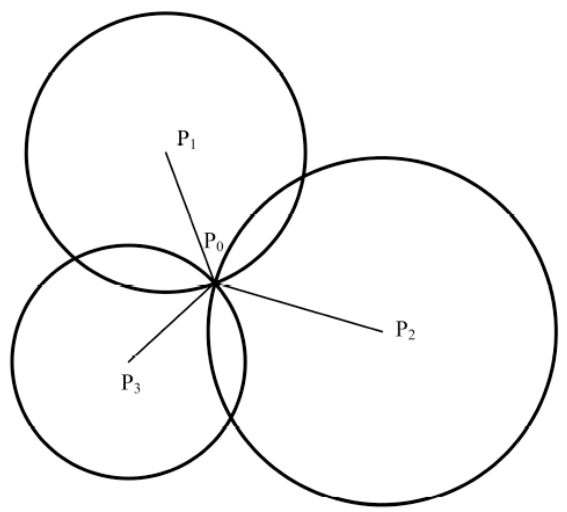

Fig. (2). Ideal model of trilateration measurement. 
However, in the actual application environments, the random variables caused by the presence of noise during the distance measurement process make the unknown node located in a region (see Fig. 3), the centroid of which can be viewed as the unknown node position.

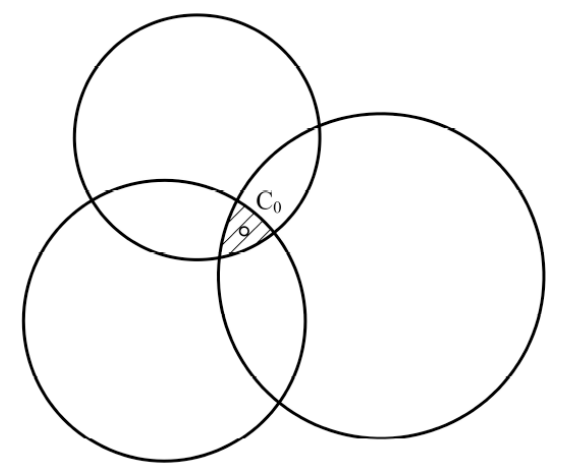

Fig. (3). The centroid of trilateration measurement in the actual application environments.

The weighted centroid localization idea is to introduce the distance between the unknown node and anchor node as a weighted factor, and the distance is inversely proportional to the factor, which reflects influence degree of the anchor node to the position of the centroid. When the unknown node receives the $\mathrm{k}(\mathrm{k}>=3)$ anchor node signals, they can constitute up to $\mathrm{c}^{3}{ }_{\mathrm{k}}$ triangles and approximate $\mathrm{c}^{3}{ }_{\mathrm{k}}$ coordinates of the unknown node can be calculated by using the weighted centroid calculation. The final position of the unknown node can be obtained through the second weighted centroid computation.

\subsection{The Weighted Centroid Computation in Trilatera- tion Measurement Method}

It's assumed that the unknown node $p_{0}$ carries out trilateration measurement by receiving the information from the anchor nodes $p_{1}, p_{2}, p_{i}$, as shown in (Fig. 4).

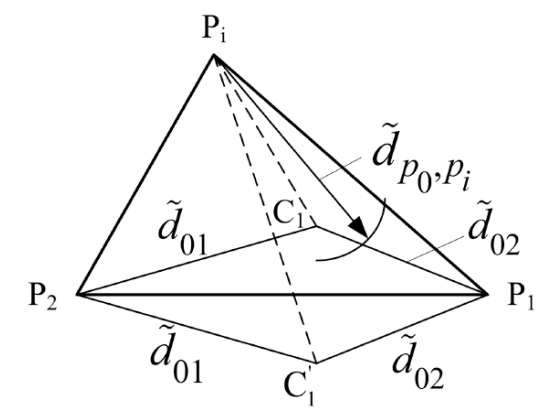

Fig. (4). The Weighted Centroid Computation in Trilateration Measurement.

According to the distance $\tilde{d}_{01}$ (measured between $p_{0}$ and $p_{1}$ ) and the distance $\tilde{d}_{02}$ (measured between $p_{0}$ and $\left.p_{2}\right)$, combined with the coordinates of $p_{1}$ and $p_{2}$, two positions coordinates $\left(\mathrm{c}_{1}\right.$ and $\left.\mathrm{c}_{1}^{\prime}\right)$ of ${ }_{p_{0}}$ can be obtained. After the distance $d_{c 1, p i}$ between $\mathrm{c}_{1}$ and $p_{i}$ and the distance $\mathrm{d}_{\mathrm{c}_{1}, \mathrm{p}_{\mathrm{i}}}$ between $c_{1}^{\prime}$ and $p_{i}$ are calculated respectively, compared with the ranging between $p_{0}$ and $p_{i}$, the close degree for $d_{c 1, p i}$ and $\mathrm{d}_{\mathrm{c}_{1}^{\prime}, \mathrm{p}_{i}}$ to $\tilde{d}_{0 i}$ is determined, i.e., $\mathrm{c}_{1}\left(\mathrm{x}_{1}, \mathrm{y}_{1}\right)$ corresponding to the smaller value of $\left|1-\tilde{\mathrm{d}}_{\mathrm{p}_{0}, \mathrm{p}_{\mathrm{i}}} / \mathrm{d}_{\mathrm{c}_{1}, \mathrm{p}_{\mathrm{i}}}\right|<\left|1-\tilde{\mathrm{d}}_{\mathrm{p}_{0}, \mathrm{p}_{\mathrm{i}}} / \mathrm{d}_{\mathrm{c}_{1}, \mathrm{p}_{\mathrm{i}}}\right|$ can be taken as the approximate position of the unknown node. Similarly, by ranging from $p_{1}$ and $p_{i}$ to $p_{0}$ as well as proximity judgment principle, the second estimated location $\mathrm{c}_{2}$ of $p_{0}$ can be obtained; and by ranging from $p_{2}$ and $p_{i}$ to $p_{0}$ as well as proximity judgment principle, the third estimated location $\mathrm{c}_{3}$ of $p_{0}$ can be obtained.

Taking advantage of the three estimated positions $\mathrm{c}_{1}\left(\hat{\mathrm{x}}_{1}, \hat{\mathrm{y}}_{1}\right), \mathrm{c}_{2}\left(\hat{\mathrm{x}}_{2}, \hat{\mathrm{y}}_{2}\right), \mathrm{c}_{3}\left(\hat{\mathrm{x}}_{3}, \hat{\mathrm{y}}_{3}\right)$ of $p_{0}$, the weighted factors reflecting the influence degree of anchor nodes are introduced:

$$
\begin{aligned}
& w_{1}=\frac{1 /\left(\tilde{d}_{01}+\tilde{d}_{02}\right)}{1 /\left(\tilde{d}_{01}+\tilde{d}_{02}\right)+1 /\left(\tilde{d}_{01}+\tilde{d}_{0 i}\right)+1 /\left(\tilde{d}_{02}+\tilde{d}_{0 i}\right)} \\
& w_{2}=\frac{1 /\left(\tilde{d}_{01}+\tilde{d}_{0 i}\right)}{1 /\left(\tilde{d}_{01}+\tilde{d}_{02}\right)+1 /\left(\tilde{d}_{01}+\tilde{d}_{0 i}\right)+1 /\left(\tilde{d}_{02}+\tilde{d}_{0 i}\right)} \\
& w_{3}=\frac{1 /\left(\tilde{d}_{02}+\tilde{d}_{0 i}\right)}{1 /\left(\tilde{d}_{01}+\tilde{d}_{02}\right)+1 /\left(\tilde{d}_{01}+\tilde{d}_{0 i}\right)+1 /\left(\tilde{d}_{02}+\tilde{d}_{0 i}\right)}
\end{aligned}
$$

Using the weighted centroid calculation through the anchor nodes $p_{1}, p_{2}, p_{i}$, the final estimated position of the unknown node $p_{0}$ can be obtained: $\hat{\mathrm{x}}_{\mathrm{i}}=w_{1} \hat{\mathrm{x}}_{1}+w_{2} \hat{\mathrm{x}}_{2}+w_{3} \hat{\mathrm{x}}_{3}$, $\hat{\mathrm{y}}_{\mathrm{i}}=w_{1} \hat{\mathrm{y}}_{1}+w_{2} \hat{\mathrm{y}}_{2}+w_{3} \hat{\mathrm{y}}_{3}$. When solving the weighted factors in DGC-TWCL algorithm, the estimated value $\hat{d}_{0 i}$ is adopted after optimization and correction.

\subsection{Twice-weighted Centroid Computation}

As described in Section 2.2, when the unknown node $p_{0}$ has received ranging information from $p_{1}, \ldots, p_{r}$, the distances of the estimated position $\hat{d}_{01}, \hat{d}_{02}, \ldots, \hat{d}_{0 r}$ between the unknown node and anchor nodes can be obtained by distance geometry constraints optimization of Cayley-Menger determinant. Consider the following set $\left\{p_{1}, p_{2}, p_{3}\right\},\left\{p_{1}, p_{2}, p_{4}\right\}, \ldots,\left\{p_{1}, p_{2}, p_{r}\right\}$ in which each element consists of three anchor nodes, the weighted centroids collection $\left\{O_{1}\left(\hat{x}_{1}, \hat{y}_{1}\right), O_{2}\left(\hat{x}_{2}, \hat{y}_{2}\right), \ldots, O_{r-2}\left(\hat{x}_{r-2}, \hat{y}_{r-2}\right)\right\}$ can be calculated respectively through the trilateration measurement method and the final unknown node position can be obtained by using the second weighted centroid computation as below:

$$
\hat{x}=\frac{\sum_{k=3}^{r}\left[\hat{x}_{k-2} /\left(\hat{d}_{01}+\hat{d}_{02}+\hat{d}_{0 k}\right)\right]}{\sum_{k=3}^{r}\left[1 /\left(\hat{d}_{01}+\hat{d}_{02}+\hat{d}_{0 k}\right)\right]}
$$




$$
\hat{y}=\frac{\sum_{k=3}^{r}\left[\hat{y}_{k-2} /\left(\hat{d}_{01}+\hat{d}_{02}+\hat{d}_{0 k}\right)\right]}{\sum_{k=3}^{r}\left[1 /\left(\hat{d}_{01}+\hat{d}_{02}+\hat{d}_{0 k}\right)\right]}
$$

\section{DGC-TWCL ALGORITHM DESCRIPTION AND EXPERIMENT ANALYSIS}

\subsection{Algorithm Description}

The DGC-TWCL algorithm includes two parts, one is solving the ranging optimization value provided by distance geometry constraints(DGC) of Cayley-Menger determinant, and the other is twice weighted centroid (TWC) computation.

The DGC portion is described as follows:

Step 1: After the unknown node $p_{0}$ has received ranging information from the known anchor nodes $p_{1}, \ldots, p_{r}$, the sequence $\tilde{d}_{01}<\tilde{d}_{02}<\cdots<\tilde{d}_{0 r}$ with descending order is set. Let the ranging errors be $\varepsilon_{1}, \varepsilon_{2}, \ldots, \varepsilon_{r}$;

Step 2: Taking $p_{0}$ and $\left\{p_{1}, p_{2}, p_{i}\right\}, i=3, \ldots, r$ into the CayleyMenger determinant $D\left(p_{0}, p_{1}, p_{2}, p_{i}\right)=0$, the function $f_{i}\left(\varepsilon_{1}, \varepsilon_{2}, \varepsilon_{i}\right)=0$ is determined whose variables are $\varepsilon_{1}, \varepsilon_{2}, \varepsilon_{i}$;

Step 3: Taking the minimum value of $J=\varepsilon_{1}^{2}+\varepsilon_{2}^{2}+\ldots+\varepsilon_{r}^{2}$ as objective function, $r-2$ equations from Step 2 as constraints conditions, nonlinear optimization solutions $\varepsilon_{1}^{*}, \varepsilon_{2}^{*}, \ldots, \varepsilon_{r}^{*}$ are obtained;

Step 4: According to $\hat{d}_{0 i}=\sqrt{\tilde{d}_{0 i}^{2}-\varepsilon_{i}^{*}}, i=1,2, \ldots, r$, the estimated position of the unknown node to the anchor node can be obtained;

The TWC part is described as follows:

Step 5: by using $\left\{p_{1}, p_{2}, p_{i}\right\}, i=3, \ldots, r$ to constitute a collection of $r-2$ triangles, in accordance with the method in Section 3, three position coordinates can be calculated by trilateration measurement and proximity principle in each triangle, and then the weighted centroid position coordinates can be obtained by making use of the factors $w_{1}, w_{2}, w_{i}$ inversely proportional to the estimated distance value $\hat{d}_{0 i}$ within each triangle;

Step 6: By adopting the method in Section 3.2 and introducing the second centroid weighted factors, the final estimated position coordinate $p_{0}(\hat{x}, \hat{y})$ of the unknown node $p_{0}$ can be obtained.

\subsection{Experiment Analysis}

Against the DGC-TWCL positioning method proposed in this paper, the positioning error, positioning error sample variance, the scalability and robustness of the algorithm have been evaluated. Experiment simulation scene has been set in a rectangular area of $8 \mathrm{~m} * 8 \mathrm{~m}$. Consider positioning of the unknown node $p_{i}$ located at the grid point under the configuration cases of three, four and five anchor nodes respectively. Suppose the ranging error is within $\pm 10 \%$ random value of the true distance. When three anchor nodes $\left\{b_{1}, b_{2}, b_{3}\right\}$ are placed in the points of $(4,8),(0,0),(8,0)$ (see Fig. 5), the testing nodes set is $\left\{\mathrm{p}_{1}, \mathrm{p}_{2}, \ldots, \mathrm{p}_{22}\right\}$. The four anchor nodes configuration is shown in (Fig. 6) and the testing nodes set is $\left\{\mathrm{p}_{1}, \mathrm{p}_{2}, \mathrm{p}_{6}, \mathrm{p}_{7}, \mathrm{p}_{8}, \mathrm{p}_{10}, \mathrm{p}_{11}, \mathrm{p}_{13}, \mathrm{p}_{14}, \mathrm{p}_{16}, \mathrm{p}_{17}, \mathrm{p}_{19}, \mathrm{p}_{20}\right\}$. The five anchor nodes configuration is shown in (Fig. 7), and the test nodes set is $\left\{\mathrm{p}_{1}, \mathrm{p}_{2}, \mathrm{p}_{4}, \mathrm{p}_{6}, \mathrm{p}_{7}, \mathrm{p}_{10}, \mathrm{p}_{12}\right\}$.

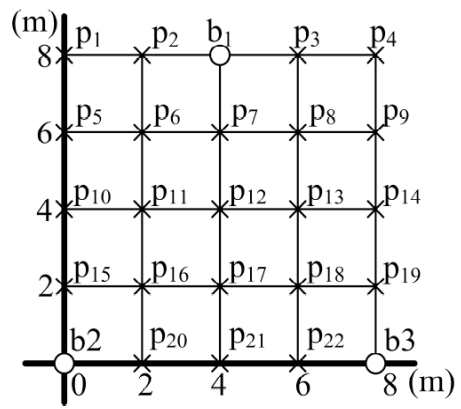

Fig. (5). Experiment simulation scene with three anchor nodes in DGC-TWC.

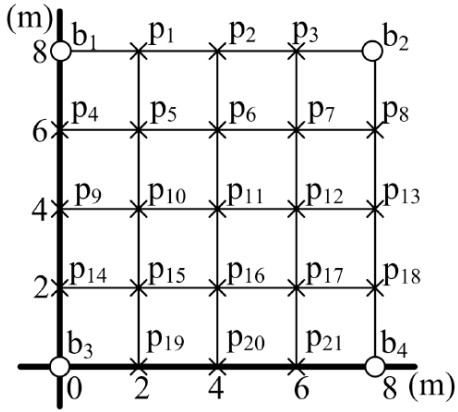

Fig. (6). Experiment simulation scene with four anchor nodes in DGC-TWC.

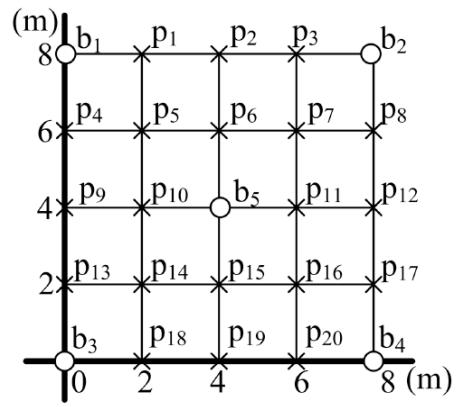

Fig. (7). Experiment simulation scene with five anchor nodes in DGC-TWC.

Besides, the positioning states of the linear least squares(LLS) estimation, mean centroid and weighted centroid location are calculated under the same ranging condition. The calculated indicators include the biggest error(BE), the smallest error(SE), the mean error(ME) and the error sample variance $(\mathrm{SV})$, where $e_{i}=\sqrt{\left(\hat{x}_{i}-x\right)^{2}+\left(\hat{y}_{i}-y\right)^{2}}, i=1,2, \ldots, n$ is one point error in the testing nodes set, and $B E=\max \left\{e_{i}\right\}$, $S E=\min \left\{e_{i}\right\}, M E=\left(\sum_{i=1}^{n} e_{i}\right) / n, S V=\frac{1}{n-1} \sum_{i=1}^{n}\left(e_{i}-\bar{e}\right) \cdot \bar{e}$ is the averaged 
measurement error of the testing nodes set. Ranging error optimization process takes advantage of fsolve in Matlab optimization toolbox. The experiment results with three, four and five anchor nodes are shown in (Table 1, Table $\mathbf{2}$ and Table 3) respectively. The corresponding graphic plotting according to the data from (Table $\mathbf{1}$ to Table $\mathbf{3}$ ) is shown in (Fig. 8).

As it can be seen from Table $\mathbf{1}$ to Table $\mathbf{3}$ and Fig. (8), under the three anchor nodes configuration, the smaller smallest error(SE) can be obtained in DGC-TWCL with respect to the other positioning methods. On the aspect of the mean error (ME), except to obtain a value slightly larger than the mean centroid under five anchor nodes configuration, the other values are lower than the other three positioning algorithm. Compared with the indicators of the biggest error(BE) and sample variance(SV), the values in DGC-TWCL are medium, which shows that distance fluctuation degree between the positioning result and true position of the algorithm is moderate. In addition, under the three anchor node configuration program, the performance of the above four statistical indicators is stable in DGC-TWCL, which indicates the scalability and robustness of the algorithm. On the other hand, DGC-TWCL algorithm increases the calculation amount and time because of the ranging calibration and the twice weighted factors computation process, which is more suitable for the positioning occasions of centralized singlehop and medium anchor nodes density. Table 4 shows the comparison of the several positioning algorithms in computation overhead, positioning accuracy, stability and scalability. Wherein $r$ is the number of anchor nodes, $T_{\text {inv }}$ is the time-consuming for calculating the generalized inverse matrix in LLS algorithm, $T_{\mathrm{cl}}$ is the time overhead for seeking the centroid of one trilateration measurement, $\mathrm{T}_{\mathrm{w}}$ is the time consumption for computing the weighted factors and $T_{g}$ is the time spent on the measurement correction. By increasing a certain amount of calculation seen from Table 4, the positioning accuracy and stability in DGC-TWCL can be improved.

Table 1. Indicators Statistics of Experiment Results Under 3 Anchor Nodes

\begin{tabular}{|c|c|c|c|c|}
\hline \multirow{2}{*}{ Localization Algorithm } & \multicolumn{4}{|c|}{3 Anchor Nodes } \\
\hline & BE & SE & ME & SV \\
\hline LLS & 2.2139 & 1.2086 & 1.5443 & 0.0622 \\
\hline Mean Centroid & 3.5577 & 0.4706 & 1.5313 & 0.9767 \\
\hline Weighted Centroid & 3.4981 & 0.5147 & 1.5081 & 0.9900 \\
\hline $\begin{array}{l}\text { DGC- } \\
\text { TWCL }\end{array}$ & 3.6956 & 0.3355 & 1.4203 & 0.7920 \\
\hline
\end{tabular}

Table 2. Indicators Statistics of Experiment Results Under 4 Anchor Nodes

\begin{tabular}{|c|c|c|c|c|}
\hline \multirow{2}{*}{ Localization Algorithm } & \multicolumn{4}{|c|}{4 Anchor Nodes } \\
\hline & $\mathbf{B E}$ & SE & ME & SV \\
\hline LLS & 0.0563 & 0.0028 & 0.0288 & $0.2211 * 10^{-3}$ \\
\hline Mean Centroid & 0.1214 & 0.0026 & 0.0341 & $0.9341 * 10^{-3}$ \\
\hline Weighted Centroid & 0.0508 & 0.0025 & 0.0334 & $0.9511 * 10^{-3}$ \\
\hline $\begin{array}{l}\text { DGC- } \\
\text { TWCL }\end{array}$ & 0.0560 & 0.0018 & 0.0240 & $0.3469 * 10^{-3}$ \\
\hline
\end{tabular}

Table 3. Indicators Statistics of Experiment Results Under 5 Anchor Nodes

\begin{tabular}{|c|c|c|c|c|}
\hline Localization Algorithm & \multicolumn{4}{|c|}{4 Anchor Nodes } \\
\hline Mean Centroid & 0.1214 & 0.0026 & 0.0341 & 0.1124 \\
\hline Weighted Centroid & 0.0508 & 0.0025 & 0.0334 & 0.0531 \\
\hline
\end{tabular}



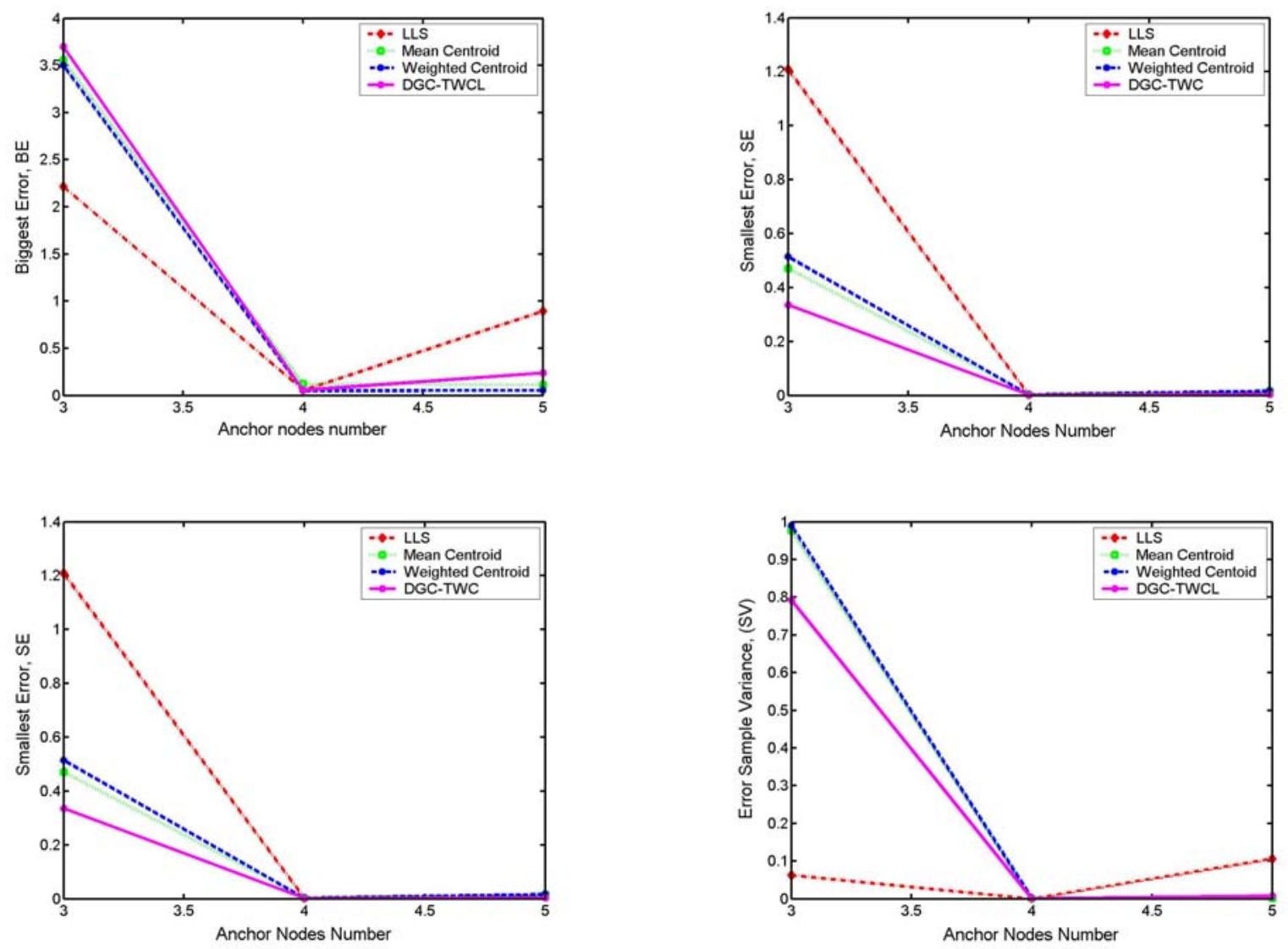

Fig. (8). The corresponding graphic plotting to experiment indicators statistics.

Table 4. Performance Comparison of Several Positioning Algorithm

\begin{tabular}{|c|c|c|c|c|}
\hline \multirow{2}{*}{ Computation Overhead } & LLS & Mean Centroid & Weighted Centroid & DGC-TWCL \\
\cline { 2 - 5 } & $\mathbf{T}_{\mathrm{inv}}$ & $(\mathbf{r}-\mathbf{2}) * \mathbf{T}_{\mathrm{cl}}$ & $\mathbf{( r - 2} * \mathbf{T}_{\mathrm{cl}}+\mathbf{T}_{\mathrm{w}}$ & Lower \\
\hline \hline Positioning Accuracy & Lower & Lower & Lower & Better \\
\hline Positioning Stability & Lower & Lower & Better \\
\hline Positioning Scalability & Better & Better & Better & Better \\
\hline
\end{tabular}

\section{CONCLUSIONS}

For node localization in wireless sensor network, because of its low-cost feature, it is impossible for each node to have the ability to directly access to the location information such as GPS global positioning system. Most nodes localization firstly makes distance measurements with anchor nodes, and due to the presence of measurement noise and estimation error, the ranging value is non-precise. In this paper, the DGC-TWCL algorithm proposed uses geometrical relationship among the nodes in wireless sensor network formed by Cayley-Menger determinant, takes the minimum of ranging squared error sum as the optimization target and corrects the non-exact distance measurement values. Combined with the twice weighted centroid calculation, the high accuracy and robustness positioning results can be achieved.

\section{CONFLICT OF INTEREST}

The author(s) confirm that this article content has no conflicts of interest.

\section{ACKNOWLEDGEMENT}

Declared none.

\section{REFERENCES}

[1] J. Blumenthal, R. Grossmann, F. Golatowski, and D. Timmermann, "Weighted Centroid Localization in Zigbee-based Sensor Networks", http://ieeexplore.ieee.org/xpl/login.jsp?tp=\&arnumber $=4447528 \&$ url $=$ http $\% 3 \mathrm{~A} \% 2 \mathrm{~F} \% 2$ Fieeexplore.iee. org $\% 2 \mathrm{Fxpls}$ $\% 2$ Fabs_all.jsp\%3Farnumber\%3D4447528. The date of access: 2013-9-10.

[2] Y. Chen, J. Francisco, W. Trappe, and R.P. Martin, "A practical approach to landmark deployment for indoor localization", IEEE SECON Proceedings, pp. 365-373, 2006. 
[3] N.B Priyantha, A. Chakraborty, H. Balakrishnan, "The cricket location-support system", Proceedings of the 6th Annual International Conference on Mobile Computing and Networking, Boston, pp. 32-43, 2000.

[4] L. Doherty, K.S.J Pister, and L.E Ghaoui, "Convex position estimation in wireless sensor networks", Proceedings of the IEEE INFOCOM, vol. 3, pp. 1655-1663, 2001.

[5] D. Nicolescu and B. Nath, "Ad-Hoc positioning systems", Proceedings of the 2001 IEEE Global Telecommunications Conference, vol. 5, pp. 2926-2931, 2001.

[6] D. Niculescu, B. Nath, "DV based positioning in ad hoc networks", Journal of Telecommunication Systems, vol. 22, no. 1/4, pp. 267$280,2003$.
[7] D. Koks, "Numerical calculations for passive geolocation scenarios", Technical Report, DSTO-RR-0000, 2005.

[8] W.A. Gardner, C.K. Chen, "Signal-selective time-difference-ofarrival estimation for passive location of man-made signal sources in highly corruptive environments", IEEE Transactions on Signal Processing, vol. 40, no. 5, pp. 1168-1184, 1992.

[9] M. Cao, B.D.O. Andersonb, and A.S. Morsea, "Sensor network localization with imprecise distances", System and Control Letters, vol. 55, no. 5, pp. 887-893, 2006.

[10] G. Mao, B. Fidan, and B.D.O. Anderson, "Wireless sensor network localization techniques", Computer Networks, vol. 51, no.1, pp. 2529-2553, 2007.

Received: August 13, 2013

(C) Gao and Peng; Licensee Bentham Open.

This is an open access article licensed under the terms of the Creative Commons Attribution Non-Commercial License (http://creativecommons.org/licenses/by-nc/3.0/) which permits unrestricted, non-commercial use, distribution and reproduction in any medium, provided the work is properly cited. 